\title{
ON THE BALANCE OF PRESSURE WITHIN THE SKULL.
}

\section{BY JAMES OAPPIE, M.D.}

IT being admitted that the brain is the organ through which mental acts or states are more immediately produced or revealed, the working condition of the former becomes the correlative of the existence of sensation, memory, thought, and volition. The working condition of the brain depends on a certain standard of structare, and a certain relation to the circulation and to other structures and agents. The more that structure and these various relationships are understood, the better qualified shall we be to trace how far any given mental act or state is related to any special sphere or mode of activity in the nervous centre, and vice versed.

In studying the causation of mental phenomena we need not be deterred by the fact that our knowledge, at the best, is likely to be always remote. It may not be the less positive so far as it may pretend to go. We must ever be content to accept the fact of conscionsness as nltimate. The physiologist will never be able to perceive why the brain's activity should be associated with its manifestations. Yet, if a correlation be assumed, he may be able to determine why one form of consciousness rather than another is present; why it is present with a felt amount of intensity; or why in certain circumstances it is obscured or suspended. The nexus between molecular activity of brain and activity of thought and volition may for ever remain "unthinkable," but many of the modifying conditions of the molecular activity may certainly be determined, and the remote but necessary influence of these on the mode and intensity of mental action may thus to some extent be recognised. 
In the present essay I wish to give prominence to some of the more general conditions in which the brain is placed-to trace a relation between the organs' mode of working and certain peculiarities in its physical surroundings. I shall have no experiments to detail, nor anything new to reveal in regard to structure. I shall simply apply well-understood principles in physics toward explaining some facts in encephalic physiology, and shall have to depend for the correctness of conclusions on the appositeness of the anelogies adduced, and the coherence of my argument. I am aware that in these days of physiological laboratories, when every inference has to be tested by an appeal to the senses, such a plan may not be allowed a high place in the methods of exact science. But in addition to the fact that it is only to a limited extent the general practitioner can avail himself of the more exact instruments of research and experiment to advance physiology, it is possible that some subjects may, at least up to a certain point, be quite successfully treated by broad views being taken of general relationships. In every phenomenon there is of necessity a chain of cansation, but the revelations of minute analysis are too frequently only isolated links. These furnish interesting facts rather then explanations. No analysis of sea water would ever enable us to explain the ebb and flow of the tides. It may be, therefore, that by holding some vital phenomena at arm's length, and taking \& bird's-eye view of palpable relations, the order of combination and sequence which constitutes causation may be recognised more readily than by atomic or microscopic analysis of the instruments concerned in their production.

It is becoming trite to remark that in the development and activity of everything living, its " environment," "surroundings," or "medium" has an influence only second in importance to that of special structure.

The physiological bearing of the brain's surroundings does not seem to have received the attention it deserves. The skull has been regarded simply as an organ of protection, and - less importance is now attached to the peculiarities of the encephalic circulation than was the case many years ngo.

Restricting ourselves in the meantime to the skull, much 
has been written to show how well adapted its structure is to serve as an organ of protection. By its form, and by the firmness and elasticity of its walls, it combines moderate weight with great power of resistance, and thus, while not a burden to the individual, it secures the delicate structure of the brain against physical injuries.

It is more than probable, however, that the mechanical properties of the skall must have an important effect in influencing the physiological action of the brain itself. If its osseous walls are efficient in affording resistance to agents from without, they will no less exert repression on the forces operating within its covity. The fact that there is a rigid limit to the brain's expansion cannot be without significance.

That in certain circumstances there is a decided tendency to expansion of the brain is well known. When it has been so exposed from injury of the skull that its behaviour can be observed, it has always been noticed that while during sleep the organ tends to sink or retire from the inner surface of the skull, in wakefulness it expands, and not only fills the whole cavity, but it may protrude beyond the aperture. Some forces are at work in the latter state which are quiescent or less powerful in the former. The tendency of these is to make the brain increase in bulk. If its rigid envelopes were suddenly to give way when, for example, severe muscular efforts are being made, the momentum of the blood would cause the vessels to be more completely filled, and the organ would instantly expand beyond the balk it could possibly occupy while they were entire. To any tendency of this sort, however, the walls of the skull oppose an effectual barrier.

Assuming, then, that when the brain is active its function is exerted under a state of physical repression or stress, we have to consider how such a circumstance may operate in modifying the results of activity.

In physics we have abundant examples of the behaviour of energy under a state of repression. Its tendency is to explosion; or, if liberated in a special direction, to produce intensity of discharge. The utility of the steam-engine, the rifle, and the electric telegraph depends on our being able to control the liberation of certain forces under stress. 
From the analogy of physics, then, we inay reasonably suppose that whatever energy the brain is able to produce or liberate, it must be more concentrated and sustained, more readily or more completely directed into some special channel, than if it were diffused in all directions in its very production. Or, if we look at the mental correlatives, we may infer that sensation and perception are more exact and acute; thought more coherently sustained; efforts to produce muscular movements stronger and more accurately directed, on account of the physical restraint to which the organic substratum is subjected.

Of course if the brain as a whole requires some counterpoise or fulcrum to insure a required intensity in the discharge of its energy, the same law must apply to individual portions of the brain. Each local or limited area of ganglionic structure which may have specific duties to perform must receive a background of resistance or support from other structures, and increase or failure of this support will have a modifying effect on the result.

It becomes at once obvious, then, that a knowledge of the sources of internal pressure, and of the conditions by which it is modified, is necessary to ensble us to understand an important department of encephalic physiology.

The immediate sources of pressure will be similar to those in other parts of the body. There is, in the first place, the expansive pressure of simple growth. When the molecular elements are multiplying or developing into structures more cellular, they attract, with more or less energy, materials from the neighbouring blood-vèssels; and in assimilating these an actively expansive force is brought into play, the aggregate amount of which will depend on the rapidity of the process, and the area over which it extends. It is no part of our plan, however, to take cognizance of the intracranial pressure produced from this cause, although, especially in diseased conditions, its consideration is at times of the highest importance.

The second and, so far as the present inquiry is concerned, more important source of pressure is the movement of fluids. We assume that the mechanical force so exerted is in accordance with the ordinary laws of physics. The greater the bulk 
and the more rapid the movement, the stronger will be the pressure. Applied to the circulation within the skull, this principle will be equally true in regard to individual vessels or to clusters of vessels.

From this restricted point of view as to the origin of intracranial pressure, its balance will depend simply on local alterations in the distribution of blood through the vessels. Every change in its circulation will be accompanied by a change of pressure in or on the brain.

Our next object, then, must be to determine on what conditions any alteration in the intracranial circulation may depend.

The principal factors concerned in the supply and distribution of blood through the brain are-1, the action of the heart on the general circulation; 2 , the action of the vaso-motor nerves on any of the branches of the circle of Willis; 3 , the molecular movement in the brain tissue; and 4 , the pressure of the atmosphere opposing the flow of blood from the venous sinuses.

In considering on which of these conditions local alterations within the skull occur, the action of the heart may be kept out of view. The ratio of that action will be the same to the whole cavity, and any change of impulse will equally affect all the vessels. Local modifications must be caused by local conditions.

The conditions which will most immediately affect the local distribution of blood are the calibre of the feeding vessels and the activity of the molecular movements. So much sympathy exists between these that they should be considered together. They are so intimately joined that it is impossible to say where causative influence begins or ends. If any distinction, bowever, is to be made, then greater importance must be attached to the agitation of the molecules. Nothing definite is known as to the action of the vaso-motor nerves on the arteries within the skull. The result of experiments has been negative or contradictory.' But we can conceive of the movements of the tissue-molecules acting on the circulation in

1 Dee 'Physiology and Pathology of the Sympathetio Byotem of Nerves. By Dra. Eulenburg and Guttmann. Trauslated by Dr. Napier, 'Journnl of Meutal Science,' July 1878.

VOL. II 
various ways. Their agitation may communicate its infinitely rapid waves directly to the blood; or the reaction between the blood and the tissue may so modify the attraction of the former for the capillary walls, that the sphere of greatest activity in reaction constitutes the "line of least resistance" along which fluids under pressure must of necessity tend to move; or the molecular agitation may affect the afferent nerve of a vessel-controlling ganglion, which again transmits an influence to the small vessels. It is more likely that any or all of these modes are correct than that any ganglion by itself should have some intuitive perception of the wants of the tissue. Practically, therefore, at all events in the normal state, we may also leave the action of the vaso-motor nerves ont of riew, and restrict ourselves to the influence which the molecular movement itself possesses of modifying the circulating current.

From the biological point of view, then, the molecular movement in the brain tissue and the movement of blood in the capillaries cannot be separated. Anatomically, such a separation is difficult; physiologically, it is impossible. As an organ which produces and discharges energy, the brain is as much dependent on its supply of blood as the burning fuel is dependent on a current of air : 'and, again, the flow of blood in the capillaries depends as much on activity in the molecules, as the draught of air through the burning fuel depends on the act of combustion. Check the blood-current, and the agitation of the molecules, with the function involved in their activity, is at once suspended or modified; stimulate the molecular movement, and the blood is transmitted with greater energy. The physiology which would ignore this reciprocal action takes a retrograde step of at least half a century. Even as a "working hypothesis" no amount of precision in describing changes in the histological elements of tissue would ever make up for its loss.

In regard to the influence of the atmospheric pressure in modifying the encephalic circulation, that I consider to be one of very great importance." It results from the physicel

1 Bee ' On the Relation of the Oranial Contents to the Presgure of the $\Delta$ tmasphere,' ' Edin. Med. Journal,' Aug. 1874. 
qualities of the cranium, which only permit the contents of the cavity to be affected through the blood-vessels which enter or leave the bony walls. It tends to oppose the exit of blood from the venous sinuses, and the backward pressure so occesioned will, of course, be exerted on the internal surface of the venous vessels through their whole extent. It will thus assist in preventing the block in the entire encephalic circulation that would be caused if the arterial impulse were to press the brain directly against the dura mater. The immediate physical result, as we shall afterwards more fully explain, will depend on the support the vessels receive on their external surface. If the forces causing brain-expansion be relaxed, the veins will be distended; and thus a certain amount of tension is at all times sustained in the brain tissue. On the other hand, if the brain tends to expand, some room is gained by the veins being compressed and their contents lessened.

Assuming, then, that molecular agitation is the dominant factor in modifying the movement of blood-that its intensity and sphere condition the rapidity and direction of the circulation, we are in a position to discugs some details as to the balance of pressure within the skull.

When the brain from a condition of rest, as in sleep, is roused to activity, the immediate effects are to increase the agitation in the molecular structures and to cause more rapid blood movement. This, of course, involves increased pressure within the capillaries, and the result, as we have already seen, is to cause an expansive tendency in the brain itself. To this tendency two important checks are opposed. These are, in the first place, the rigid limits presented by the cranial walls, and to which we need not further allude. The second check is the peculiar stracture of the pia mater.

This membrane presents a curious interlacement of arterial and venous vessels, the physiological meaning of which has not received the attention it deserves. Its connections with the brain are entirely capillary. The large mass of the brain is fed by arteries which from the first penetrate its substance in a state of capillary minuteness; the veins also leave the nerve tissue as capillaries, and in the pia mater they coalesce to form large tortuons vessels. Now, if expansion of the brain 
were to occur beyond a very limited extent, a process of selfstrangulation would take place. The circulation itself would be stopped. The first effect must be to compress the veins, but this compression cannot be to a greater extent than will allow whatever amount of arterial blood which enters the skull to be transmitted onwards. The result will be to increase the tension in the brain itself. The blood pressure will be expended in rapid movement.

To this tension all the fluids and solids within the skull will be alike subjected. It will not be greater at one point than at another. The area being limited and full, and so much of the contents being fluid, the important law of pressure being transmitted equally in all directions will here hold good.

A moment's consideration will enable us to perceive how such a fact is to be reconciled with the statement previously made, that every change in the intracranial circulation involves a change of pressure in or on the brain. We have simply to bear in mind the distinction to be made between active pressure and "stress," 1 or tension.

In a glass globe where gold fish are in active motion, the currents or agitation in the water can never so affect the globe itself as to make it tend to move in one direction or another. If it were fixed on wheels on a smooth table, "even were the table a lake of ice and the wheols extremely delicate, we should find that the globe would remain at rest." 2 Whether the currents in the water be strong or weak, the pressure on the sides of the vessel will be equalised through its whole oircumference. Throughout any particular level the amount of stress will be the same. But within the area of uniform stress, the disposition of movable bodies or particles will depend on the rapidity and direction of the currents, and thus, in the case before us, the movement and position of the fish may vary every moment.

So, within the skull, whether stress be stronger or weaker, it must be equalised throughout the cavity; but the direction

1 'The ward 'stress ' as an equivalent for 'action and reaction,' and as a generio name for pressure, tension, \&c, will save future generations a great deal of tronble." -Prof. Clebre Maxwell, 'Nature, Aug. 20, 1874.

- Prof. Balfour Stewart, 'Conservation of Energy,' p. 9. 
of the blood currents, and therefore the disposition of the movable solids, will be subject to infinite variation, and there will be no end to the modes in which the action of the brain itself may be modified from this cause. The law of pressure by fluids in motion-as affected by volume and velocity-must here come into play. As the blood is directed in greater quantity or at a more rapid rate through particular vessels, the active pressure will there be greater or less. Then, of course, the brain itself is not a fluid body. Its cell elements and fibres may be kept closer or more apart, according to the disposition of the fluids by which it is surrounded and saturated. As it can readily be understood that a certain compactness, or, it may be, freedom to move, may be necessary for proper functional action, the intensity or direction of the currents may, from their purely mechanical effect, stimulate, modify, or suspend activity through the whole organ, or in limited portions of its mass.

Recurring now to the case of the brain passing from the rest of sleep to the activity of wakefulness, we have to consider in what manner the intracranial pressure is modified. During sleep the brain circulation is quiet, and stress within the skull is weak; as activity sets in the molecular agitation and the increased impetus of the circulation cause the stress to be augmented. But something more than a change in the intensity of intracranial stress must take place. The balance of active pressure is also greatly altered. To show in what manner this occurs we may reverse the order in which the changes succeed one another, and see what happens as sleep comes on.

From exhaustion, or some other ceuse, the molecular movements become less energetic, and simultaneously the hold of the capillaries on the arterial blood is weakened. But there are other physical agencies at work within the skull, and the action of these cannot remain unaffected by such a change. For example, we have seen there is at all times a special stress within the veins. The combined cardiac and capillary forces urge more blood into them; the pessive resistance of the atmospheric pressure tends to keep back that blood within the skull; and, lastly, the expansive action of the brain tends to 
obliterate the vessels themselves by pressure on their sides. Of these forces, that caused by the atmospheric pressure varies least in amount. We have therefore to do with a simple problem in the composition of forces. If one or two be relaxed, the third must bear with greater effect. Thus, if the forces which cause brain-expansion act with less energy, the backward pressure in the veins must act with more decided effect. The flow of blood from them will be retarded so long as the equilibrium tends to be disturbed. When the new equilibrium is gained the vessels will be more distended than can possibly occur during a more active state of the brain.

The ame change may be expressed in another way. If the brain tends to retire from the inner sarface of the skall (and that it does so during sleep is the unanimons testimony of all who have observed its behaviour through an opening in the bone), it will exert a suetion effort on the blood in the veins. It is impossible for suction to be exerted on the cerebrospinal fluid in the spinal canal, for the contents of this cavity are also remored from the direct action of the atmospheric pressure. ${ }^{1}$ A new balance of the circulation is therefore established; less blood circulates in the capillaries of the brain, and to an exactly corresponding extent more must be held by the veins. If a survey be taken of the various conditions involved, it will be seen that this change is a physical nocessity.

If we are now allowed to assume that when the brain passes from rest to activity, or from activity to rest, a change occurs in the balance of its circulation, then, in accordance with the principle we have insisted on, the balence of active pressure within the skull must also be altered. This change of pressure will of course affect the disposition of the brain tissue, and must hare an influence on its activity of function. During wakefulness, when the arterial and capillary circulation is most active, the pressure is outwards. It is expansive, and the reaction against the craniel walls causes the stress through the whole cavity to bo increased. Nervous energy is liberated,

1 I have elsewhere ('The Causation of Sleep ') fully discused the improbability of the cerebro-spinal finid taking the place of the diminished cerebral circulation in healtby sleep. 
and the result is some psychical or motor change. During sleep the venous circulation preponderates, and the pressure is from the surface inwards. The amount of blood within the skull may remain as before; indeed, I believe that in healthy sleep it must remain almost exactly the same. But a larger proportion of it is now external to tue brain mass, and its movement there must react physically on the brain. The latter is more or less compressed. When the molecular movement began to flag, the capacity for active function was by that very fact also weakened. But this change of pressure still further interferes with functional capaeity. When it amounts to a certain degree, function is suspended--the brain for the time fails to subserve the manifestation of the higher mental states.

We thus seem to find that, in the physical changes which accompany rest-in the more sluggish molecular movement with (as a necessary consequence) lessened intracranial stress, altered balance of the circulation and altered balance of pressurowe get a physiological explanation of sleep itself. We are furnished with a mechanism which secures a rest to the brain sufficiently lengthened to allow restorative chariges to occur. No fact in brain biology has been better ascertained than that compression of the organ prodnces unconsciousness, and this first condition of sleep we find in the distended veins of the pia mater. Then, the whole agency being under the control of the molecular activity, the pressure may be graduated infinitesimally, so as to produce the lightest slumber or a state of heavy stupor. Further, if a stimulus be applied to the brain, the whole series of conditions can be changed at once. In this important respect the above theory of sleep contrasts with any other which places the cause in a change of nutrition alone, or in the action of some vaso-motor centre. We need not wait till superfluous cerebro-spinal fluid has been got rid of. The increased capillary and diminished venous circulation will be simultaneous.

The next step of our inquiry should now be to consider some questions relating to the balance of pressure through the brain itself. Assuming that the more immediate seat of active function is also the sphere of greatest vascular activity, we 
should likely find that the activity of one part of the brain may affect the function of other parts in various other ways than by direct connection through commissural fibres. In one case, function may be suspended by a derivation of blood from its vessels, as when impressions on a sensory nerve are not felt on account of the mind being absorbed in a train of thought; in another case, an increase of energy may be occasioned from the steadier fulcrum-like support afforded, as when the intensity of muscular contraction is incressed by the simultaneous action of other muscles; in a third case, the mechanical impulse of pressure may act as a direct stimulus, as when a sudden noise causes one to start, or, indeed, as may possibly occur in the cansation of voluntary motion. Into this wide field, however, we cannot enter in the meantime. 